\title{
Similarity Measure Using Hausdorff Distance in 2D Shape Recognition System
}

\author{
Rasul Enayatifar \\ Faculty of Computing \\ Universiti Teknologi Malaysia (UTM) \\ 81310, Skudai Malaysia. \\ r.enayatifar@gmail.com
}

\author{
Rosalina Abdul Salam \\ Faculty of Science \& Technology \\ Universiti Sains Islam Malaysia (USIM) \\ 71800 Nilai, Negeri Sembilan, Malaysia \\ rosalina@usim.edu.my
}

\begin{abstract}
Shape plays an important role for human to recognize an object. Shape can be recognized easily by human regardless of size, rotation or translation. In this paper a shape recognition system is presented. In this research, authors investigate late improves in shape analysis and shape recognition issues with a concentrate on significant highlights of a shape. In the proposed method, the first stage is an edge detection method based on fuzzy cellular automata. In the second stage, shapes were clustered based on the differences of degree of each angle. Finally Hausdorff method is used for determining the rate of similarities. Simulation results demonstrate the system invariance to rotation, translation and scale.
\end{abstract}

Keywords-component; Edge detection, Fuzzy cellular Automata, Hausdorff method

\section{INTRODUCTION}

Shape is an important feature in identifying object. Recently, there has been increasing interest in the shape recognition area [1-4]. Studies demonstrated that image similarity is essential for the identification of objects[4, 5]. The structure of an overall purpose artificial system effective at recognizing arbitrarily complex 3-Dl objects without human intervention continues to be a negotiable task in computer vision. The whole picture that objects within the project scope on our retinas changes as our position changes with regards to the objects. In lifestyle $3 \mathrm{D}$ objects is usually recognized regardless of the variability in object appearance brought on by the pose from the object according to the viewer. You will need to understand the way the visual system represents the objects internally, while countering the consequences belonging to the viewing conditions, and associating the several two dimension images originating through the same three dimension object.

Although several features of object shapes are utilized by the visual object recognition, the initial requirements resulting from shape categorization increase the risk for comprehension of its underlying system very complicated in contrast to that relating to other features of objects[6]. The majority of the existing methods [7-9] for pattern recognition through optical correlation happen to be developed for 2D objects, which may have played important roles in several applications for instance detection, recognition, and classifying with the images. Nevertheless the intensity shapes from those algorithms only support the gray scale data, and lose the peak distribution information belonging to the object. Particularly for those objects, which have a similar shape and various heights, it is nearly impossible to differentiate them by observing the two dimentionintensity images. However, other theorists have argued for viewpoint invariance in shape detection. Viewpointinvariance hypothesis suppose that objects might be recognized from unfamiliar or inexperienced viewpoints.

In the first thing individuals proposed technique is the side detection. In this task, an advantage detection method is used to look for shape's edge. In these studies we applied a good sharp edge detection algorithm which can be proposed by Meybodi. Then some important characteristic including variety of angles and degree of every angle will extract through the input object. Then each object is going to be clustered base on mentioned characteristics. In the end, similarity rate is determined depending on Hausdorff method $[10,11]$.The remainder of the article is organized the following: In next section, a background studies of our tasks are presented. In section 3 a new technique is proposed. In section 4 , the simulation results are presented. Section 5 contains the conclusions.

\section{PRELIMINARIES}

\section{A. Cellular Automata}

Cellular Automata (CA) are discrete systems. CA behavior seemingly depending on local relations. A cellular automaton is made up of grid of cells; all of them is within the finite variety of state $[12,13]$. In CA, plenty of time can also be discrete, and the condition of a cell can be often a function of the prior states of their neighbor cells. A uniform rule is utilized to each and every cell and its particular neighbours and every time that it is used, the newest states of cells are created [14].

\section{B. Cellular Learning Automata}

The Cellular Learning Automaton (CLA) can be a mathematical function for implementing dynamics of an complex system which contains numerous simple components. In reality, CLA can be a CA through which all cell comes with a minumum of one learning automata (LA). The LA includes a finite pair of actions as well as goal is to understand which action within this set is the perfect action. Like CA, you will find there's uniform local rule placed on the cell and depending on this rule the chosen action gets an incentive or perhaps a penalty. If the learning method is 
selected properly, the iterative procedure for interacting using the environment can lead to collection of the suitable action in each and every cell [15].

\section{Fuzzy Cellular Automata}

Fuzzy Cellular Automaton (FCA) is often a cellular automaton where fuzzy logic is utilized towards the states of cells and transition functions. There are several explanation for FCA [16]. In FCA, the states of cells are linguistic variables along with the transition procedure are fuzzy rules. The linguistic variables are determined according to our understanding of the problem. The following state of every cell is dependent upon transition function. The existing state of every cell its particular neighbours would be the input arguments with the transition procedure. The transition function can be a uniform fuzzy function that takes the membership values with the neighbor cells and calculates the valuation on Membership from the next state. The membership values of linguistic variables which represent the State of cells are employed to present the evolution of FCA throughout the process. The group of neighbor cells is uniform and fixed throughout the process.

\section{PROPOSED METHOD}

The proposed method can be performed in three phases as follows:

Phase 1: Pre-processing.

In the first step, input shape has been extracted by edge detection algorithm which is proposed in reference [12]. For the edge detection the following steps will be conducted.

For having a high quality edge, in the first step, variance feature is used to detect the initial edges of original image. After that in the second step, power function is used to remove the information of background which is not belonging to the image's edges. The mentioned power function is defined as below:

$$
F(i, j)=\sqrt{I(i, j)^{c}}, c \succ 2
$$

As it clear in the above equation, value of $\mathrm{C}$ for all pixels is higher than 2, whereas some pixel need more than others and vice versa. To adjust the exact value of $\mathrm{C}$ for each pixel, a fuzzy cellular automaton is utilized. Finally in the last stage to detect the high quality edge, cellular learning automata is applied. For more information see reference [12].

Presented algorithm in the initial stage used of statistic characteristic of their object for primary edge detection that induces adaptively because of this algorithm whatsoever internal shape. Along at the second stage FCA and CLA [12]. Figure 1 shows the input shape pre and post edge detection.

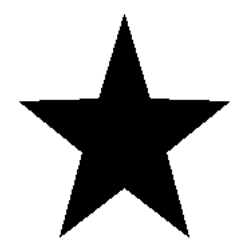

(a)

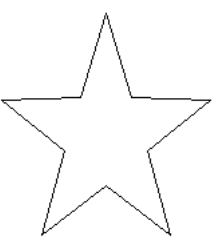

(b)
Figure 1. a) Input shape b) Edge detection

\section{Phase 2: Feature extraction}

After detecting the shape's edge, algorithm goes through the edges and the angle $(\theta)$ between three adjacent pixels is determined as follow:

$$
\theta=\arccos \frac{\overrightarrow{r_{1}} \cdot \vec{r}_{2}}{\left\|\overrightarrow{r_{1}}\right\|\left\|\overrightarrow{r_{2}}\right\|} ?
$$

Subject to:

$$
\begin{aligned}
& r_{1}=\left|x_{1}-x_{2}\right| i+\left|y_{1}-y_{2}\right| j \\
& r_{2}=\left|x_{2}-x_{3}\right| i+\left|y_{2}-y_{3}\right| j
\end{aligned}
$$

Where $x_{1}, k_{2} x_{3}$ are three sequential pixels on the shape. All angles for a specified shape are saved in the array list. The distance between two sequential angles is calculated as the length of side. Now, all shape with same number of angles is put in the same cluster.

Phase 3: Similarity

To measure the similarity of two shapes in the same cluster, we proposed the following steps:

1. Calculating the center of each $2 \mathrm{D}$ object

2. Re-scale object to set objects size.

3. Align w.r.t. position the two objects at corresponding centroids.

4. Rotate orientation to align w.r.t

After performing the above steps, Hausdorff method will be applied to measure the distance of two shapes. The Hausdorff distance $\vec{h}(\mathrm{~A}, \mathrm{~B})$ is defined as the lowest upper bound (supremum) over all points in $\mathrm{A}$ of the distances to $\mathrm{B}: \overrightarrow{\mathrm{h}}(\mathrm{A}, \mathrm{B})=\sup _{\mathrm{aeA}} \inf _{\mathrm{b} \in \mathrm{B}} \mathrm{d}(\mathrm{a}, \mathrm{b})$ with $\mathrm{d}(\mathrm{a}, \mathrm{b})$ the underlying distance, for example the Euclidean distance $L_{2}$. The Hausdorff distance H(A,B) is the maximum of $\overrightarrow{\mathrm{h}}(\mathrm{A}, \mathrm{B})$ and $\overrightarrow{\mathrm{h}}(\mathrm{A}, \mathrm{B}): \mathrm{H}(\mathrm{A}, \mathrm{B})=\max \{\overrightarrow{\mathrm{d}}(\mathrm{A}, \mathrm{B}), \overrightarrow{\mathrm{d}}(\mathrm{B}, \mathrm{A})\}$.

After finding the distance between two shapes, the following properties apply to all shapes A,B and C.

1. (Nonnegativity) $d(\mathrm{~A}, \mathrm{~B}) \geq 0$

2. (Identity) $d(A, A) \geq 0$ for all shapes $A$.

3. (Uniqueness) $d(\mathrm{~A}, \mathrm{~B})=0$ implies $\mathrm{A}=\mathrm{B}$.

4. (Strong Triangle inequality) $d(A, B)+d(A, C) i \geq d(B, C)$.

5. (Triangle inequality) $d(A, B)+d(B, C) \geq d(A, C)$

Properties (2) and (5) do not imply symmetry. Similarity measures for partial matching, giving a small distance $d(A, B)$ if a part of $A$ matches apart of $B$, in general do not obey the triangle inequality. After finding the distance between two shapes, the following properties apply to all shapes A,B and C.

6. (Nonnegativity) $d(\mathrm{~A}, \mathrm{~B}) \geq 0$

7. (Identity) $d(A, A) \geq 0$ for all shapes $A$.

8. (Uniqueness) $d(\mathrm{~A}, \mathrm{~B})=0$ implies $\mathrm{A}=\mathrm{B}$.

9. (Strong inequality) $d(A, B)+d(A, C) \geq d(B, C)$.
Triangle 
10. (Triangle inequality) $d(A, B)+d(B, C) \geq d(A, C)$

The whole process of the proposed method is shown in Figure 2.

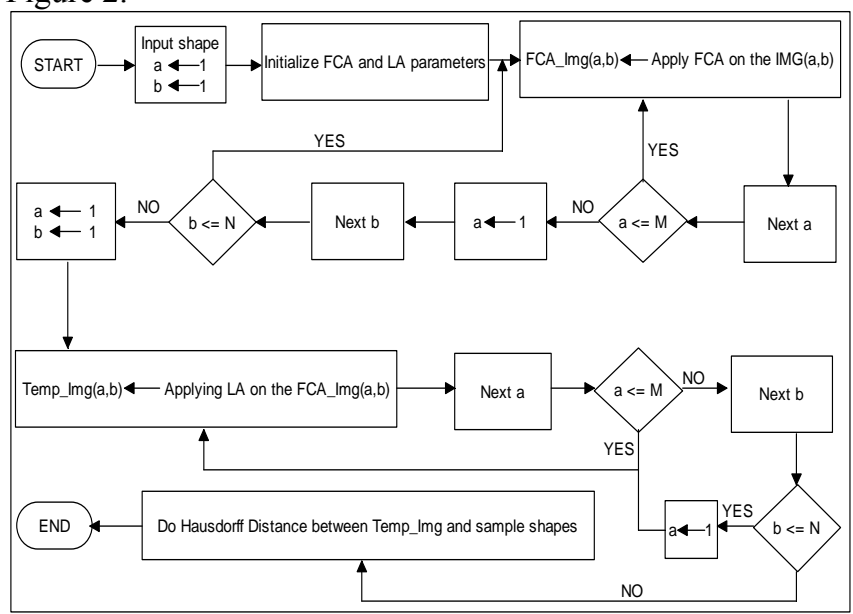

Figure 2. Overview of the Proposed Method

\section{SiMULATION RESULTS}

In this section, we examine the real performance of the proposed scheme by performing some experiments. In the first experiment, four test images (Figure 3(a,b,c,d)) have been recognized by proposed method. An existing shape Fig 3 (e) was used for comparison. Table 1 represents the similarity rate between inputting shapes and test shape.

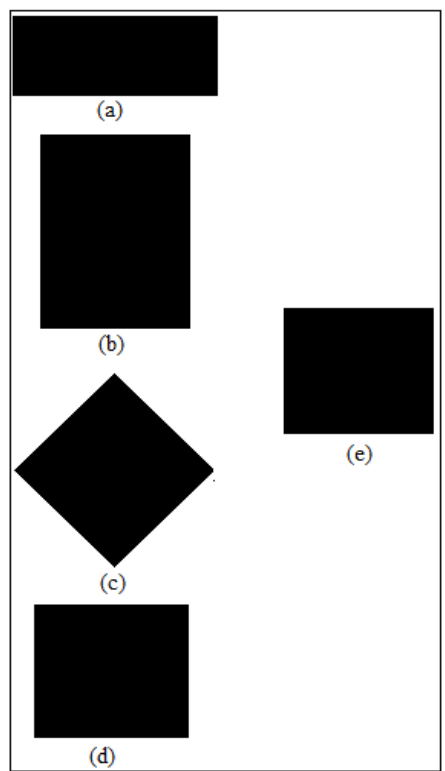

Figure 3. (a),(b),(c),(d) input shapes, (e) existing shape
TABLE 1. SIMILARITY MEASURE

\begin{tabular}{|c|c|c|c|c|}
\hline & $\begin{array}{c}\text { Figure } \\
3(\mathrm{a})\end{array}$ & $\begin{array}{c}\text { Figure } \\
3(\mathrm{~b})\end{array}$ & $\begin{array}{c}\text { Figure } \\
3(\mathrm{c})\end{array}$ & $\begin{array}{c}\text { Figure } \\
3(\mathrm{~d})\end{array}$ \\
\hline $\begin{array}{c}\text { Figure } \\
2(\mathrm{e})\end{array}$ & $71 \%$ & $73 \%$ & $96 \%$ & $96 \%$ \\
\hline
\end{tabular}

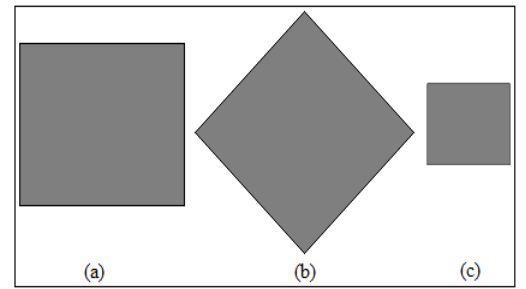

Figure 4. (a) main shape, (b) Figure $3 \mathrm{a}$ with 45 degree rotation, (c) Figure 3 a with $50 \%$ resize

TABLE 2. THE SIMILARITY RATE BETWEEN SHAPES IN FIGURE5 AND FIGURE 6

\begin{tabular}{|c|c|c|c|c|}
\hline & Figure6a & Figure6b & Figure6c & Figure6d \\
\hline Figure 5a & 0 & 0 & $78 \%$ & 0 \\
\hline Figure 5b & $91 \%$ & 0 & 0 & 0 \\
\hline Figure 5c & $68 \%$ & 0 & 0 & 0 \\
\hline Figure 5d & 0 & $74 \%$ & 0 & 0 \\
\hline Figure 5e & $70 \%$ & 0 & 0 & 0 \\
\hline Figure 5f & 0 & 0 & $81 \%$ & 0 \\
\hline Figure 5g & 0 & 0 & 0 & $\% 64$ \\
\hline Figure 5h & 0 & $73 \%$ & 0 & 0 \\
\hline Figure 5i & $63 \%$ & 0 & 0 & 0 \\
\hline Figure 5j & 0 & $82 \%$ & 0 & 0 \\
\hline Figure 5k & 0 & 0 & $66 \%$ & 0 \\
\hline Figure 5L & 0 & 0 & 0 & $61 \%$ \\
\hline
\end{tabular}




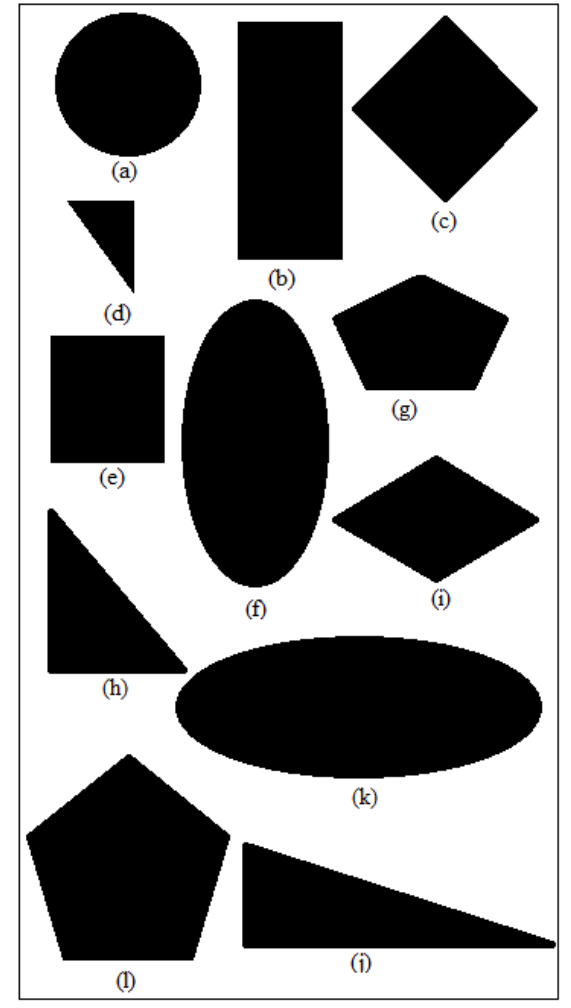

Figure 5. Sample images

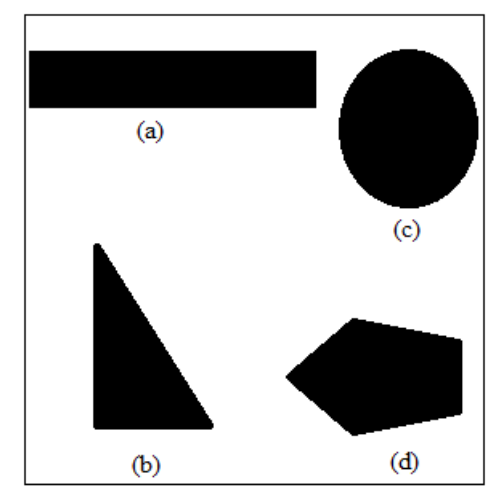

Figure 6. Test images

\section{IV.CONCLUSION}

In this paper a new method based on FCA, feature extraction and Hausdorff method is proposed. This method using FCA because of its ability to determining the thin and clear edge. Number of angle and degree of each angle are determined and shapes with the same number of angles have been put in the same cluster. In the final stage of the proposed method Hausdorff method is employed to explain the distance between to shapes in the same cluster. Simulation results shows the proposed method is resist against rotation and resize.

\section{ACKNOWLEDGMENT}

This paper presents work that are funded by Universiti Sains Islam Malaysia (USIM), Malaysia under the internal funding (PPP/FST-06-11311).

\section{REFERENCES}

[1] T. TSUJimura, Y. Minato, K. IZUMI, Shape RECOGNITION OF LASER BEAM TRACE FOR HUMAN-ROBOT INTERFACE, PATTERN RECOGNITION LETTERS, 34 (2013) 1928-1935.

[2] Y.-L. Lee, M. Lind, N. Bingham, G.P. Bingham, OBject RECOGNITION USING METRIC SHAPE, VISION RESEARCH, 69 (2012) 2331.

[3] R.-X. Hu, W. Jia, D. Zhang, J. Gui, L.-T. Song, Hand Shape RECOGNITION BASED ON COHERENT DISTANCE SHAPE CONTEXTS, PATTERN RECOGNITION, 45 (2012) 3348-3359.

[4] J. AlmazÁn, A. Fornés, E. Valveny, A NON-RIGID ApPearance MODEL FOR SHAPE DESCRIPTION AND RECOGNITION, PATTERN RECOGNITION, 45 (2012) 3105-3113.

[5] E. GonZÁlez, A. AdÁN, V. Feliú, 2D Shape REPRESEnTATION AND SIMILARITY MEASUREMENT FOR 3D RECOGNITION PROBLEMS: AN EXPERIMENTAL ANALYSIS, PATTERN RECOGNITION LETTERS, 33 (2012) 199-217.

[6] G. WANG, J. BINGO, THREE-DIMENSIONAL OBJECT RECOGNITION LEARNING ALTERS AN EARLY ERP COMPONENT OF N1, CLINICAL NEUROPHYSIOLOGY, 121 (2010) 1473-1480.

[7] A. Bhagati, N.K. Nishchal, A.K. Gupta, B.P. Tyagi, WaveletMODIFIED FRINGE-ADJUSTED JOINT TRANSFORM CORRELATOR, OPTICS \& LASER TECHNOLOGY, 40 (2008) 99-112.

[8] K. Chalasinska-Macukow, C. Gorecki, Optoelectronic IMPLEMENTATION OF THE QUASI-PHASE CORRELATOR, OPTICS COMMUNICATIONS, 93 (1992) 11-18.

[9] Y. WANG, X. Su, Y. Dou, A fast three-Dimensional obJect RECOGNITION BASED ON MODULATION ANALYSIS, OPTICS AND LASERS IN ENGINEERING, 48 (2010) 1027-1033.

[10] R.C. VeltKamp, Shape MatChING: SIMILARITY MEASURES AND ALGORITHMS, INTERNATIONAL CONFERENCE ON SHAPE MODELING AND APPLICATIONS, (2001) 188 - 197.

[11] F. Aydin AKgun, B.E. RhoAdes, Properties of SOME QHAUSDORFF MATRICES, APPLIED MATHEMATICS AND COMPUTATION, 219 (2013) 7392-7397.

[12] M.R.E. Meybodi, R., AdAPtive edge detection VIA IMAge STATISTIC FEATURES AND HYBRID MODEL OF FUZZY CELLULAR AUTOMATA AND CELLULAR LEARNING AUTOMATA, INTERNATIONAL CONFERENCE ON INFORMATION AND MULTIMEDIA TECHNOLOGY, (2009) 273-278.

[13] M. Alirezanejad, R. Enayatifar, Steganography By USING Logistic Map Function and Cellular Automata, Research JOURNAL OF APPLIED SCIENCES, ENGINEERING AND TECHNOLOGY, 4 (2012) 4991-4995

[14] S. WOLFRAM, THEORY AND APPLICATIONS OF CELlular AutOMATA, ADVANCED SERIES ON COMPLEX SYSTEMS: SINGAPORE: WORLD SCIENTIFIC PUBLICATION, (1986)

[15] M.R. Meybodi, H. Beigy, M. Taherkhani, Cellular Learning AutomataUniversity, ProceEDINGS OF 6TH ANNUAL CSI COMPUTER CONFERENCE, ISFAHAN, 2001, PP. 153-163.

[16] S. SADEGHI, A. REZVANIAN, E. KAMRANI, AN EFFicIENT METHOD FOR IMPULSE NOISE REDUCTION FROM IMAGES USING FUZZY CELLULAR AUTOMATA, AEU - INTERNATIONAL JOURNAL OF ELECTRONICS AND COMMUNICATIONS, 66 (2012) 772-779. 